\title{
A Comparison of One Hour versus Two-Hour Postprandial Glycemic Control during Pregnancy: A Prospective Cohort Study Carried out at Antenatal Ward Teaching Hospital Peradeniya, Sri Lanka
}

\author{
W. M. M. P. B. Wanasinghe ${ }^{*}{ }^{(1)}$, R. M. C. J. Ratnayake2 ${ }^{(1)}$ \\ ${ }^{1}$ Teaching Hospital, Peradeniya, Sri Lanka \\ ${ }^{2}$ Department of Obstetrics and Gynecology Faculty of Medicine, University of Peradeniya, Peradeniya, Sri Lanka \\ Email: *m178.wanasinghe@gmail.com, chathura.ratnayake@gmail.com
}

How to cite this paper: Wanasinghe, W.M.M.P.B. and Ratnayake, R.M.C.J. (2021) A Comparison of One Hour versus TwoHour Postprandial Glycemic Control during Pregnancy: A Prospective Cohort Study Carried out at Antenatal Ward Teaching Hospital Peradeniya, Sri Lanka. Advances in Reproductive Sciences, 9, 69-80.

https://doi.org/10.4236/arsci.2021.91008

Received: November 30, 2020

Accepted: January 1, 2021

Published: January 4, 2021

Copyright $\odot 2021$ by author(s) and Scientific Research Publishing Inc. This work is licensed under the Creative Commons Attribution International License (CC BY 4.0).

http://creativecommons.org/licenses/by/4.0/

\begin{abstract}
Background It is well known that both pre-excising and gestational diabetes mellitus in pregnancy is associated with multiple maternal and fetal complications. There are various guidelines developed to reduce these complications, yet there is no universal agreement regarding the ideal management. For those with pre-exciting or gestational diabetes mellitus, the control of blood sugar is assessed either with 1st-hour post-prandial blood sugar values or with the 2nd-hour post-prandial blood sugar values. Objective To compare the long-term blood glucose control (HbAlc values) of pregnant women by utilizing results of 1-hour postprandial blood sugar values against 2 -hour postprandial blood sugar values, in the antenatal ward at the professorial unit, Teaching Hospital Peradeniya. Materials and Methods A prospective cohort study was carried out among 138 pregnant women with diabetes mellitus, who were managed in two different clinics at the professorial unit, Teaching Hospital Peradeniya. The blood glucose control in group $1(n=69)$ was assessed and optimized with 1st-hour postprandial blood sugar values and group $2(n=69)$ was evaluated with 2 nd-hour postprandial values throughout the pregnancies. Following delivery, all subjects were reassessed with blood $\mathrm{HbA1c}$ and analyzed with the independent $\mathrm{t}$-test. Feto-maternal complications were analyzed and evaluated using descriptive statistics and Chi-Square tests. Results Of the subjects, 97.1\% were diagnosed with gestational diabetes mellitus. The mean value of HbA1c for group 1, managed with 1st-hour blood glucose values was 5.73 and $6.04(\mathrm{p}=0.02)$ respectively for group 2 . Fetal
\end{abstract}


macrosomia was also significantly $(\mathrm{p}<0.01)$ low in the 1 -hour blood sugar control group. Conclusion Long-term control of blood sugar was significantly superior among the subjects managed with 1st-hour postprandial blood sugars with a statistically significant reduction in foetal macrosomia.

\section{Keywords}

Gestational, Diabetes, Fetal Macrosomia, Pre-Eclampsia

\section{Introduction}

Diabetes during pregnancy (pregestational or gestational) is a leading cause of maternal and fetal complications during pregnancy [1]. Dietary advice, self-monitoring of blood glucose and insulin therapy have shown to reduce adverse perinatal outcomes in mothers diagnosed with diabetes at $24^{\text {th }}-28^{\text {th }}$ week of gestation [2] [3].

Glycemic target recommendations for the management of pregnant women with diabetes from international professional organisations for maternal glycaemic control vary widely. Various methods of glucose monitoring such as fasting, pre-prandial, postprandial, and mean 24-hour blood glucose concentrations are suggested. However, the best timing of the postprandial measurements, that is, 1-hour postprandial blood sugar control with a target value of $<7.8 \mathrm{mmol} / \mathrm{l}^{1}$ or 2 -hour control with a target value of $<6.4 \mathrm{mmol} / \mathrm{l}^{1}$ after meals is yet to be established. Therefore, this study was undertaken to assess whether the timing of measurements concerning postprandial blood sugar control has an effect on glycaemic control and to discover which postprandial glucose measurement (1-hour postprandial blood sugar control or 2-hour control) is associated with better HbA1c values, in turn, the glycaemic control of women.

A study similar by Weisz et al. 2005 has shown no significant difference between the two times of measuring glucose on glycaemic control indicated by HbA1c or perinatal outcomes [4]. However, they have taken the values of capillary post-meal glucose levels using glucometers and the procedure under different environmental conditions. In our study, the post-meal plasma glucose values were assessed with a more standardized manner and more accurate indications of exact post-meal values were taken into account. Weisz et al. have also indicated that patients managed with 2-hour glucose required more frequent insulin therapy [4]. The tight glycaemic control and reduced incidence of complications are a fact. But in the above study, perinatal outcomes weren't significantly different in the two groups managed with 2-hour post-meal capillary glucose values who had undergone strict glycaemic control with insulin. Because of variations in the methodologies used to measure glucose concentrations self-monitoring blood glucose measuring devices (Capillary blood), continuous blood glucose measuring devices (interstitial fluid glucose levels) and laboratory analysis (Venous plasma) results of the many studies are difficult to be compared. Thus, this study in- 
tended to recruit more subjects with standard laboratory analytic methods.

The neonatal complications, including hypoglycemia, hyperbilirubinemia, and respiratory compromise have been described in infants born to women with gestational diabetes, particularly with poor glycemic control [5], this further emphasizes the need of optimal glycemic control during pregnancy. A study about the number of abnormal plasma Glucose Tolerance Test values and feto-maternal outcomes has concluded that increasing number of abnormal values is associated with feto-maternal outcomes in diabetic pregnancy [6].

Therefore, this study is designed to add further evidence concerning optimal glycemic control during pregnancy in a Sri Lankan-Southeast Asian setting, where this has not been studied previously.

\section{Materials and Methods}

This analysis was conducted as a prospective cohort study at the professorial unit, antenatal ward of the Teaching Hospital, Peradeniya from June 2017 to December 2017. An ethical clearance (2017/EC/19) was obtained from the Ethical Review Committee, Faculty of Medicine, University of Peradeniya. The minimum sample size was 138 subjects with a confidence interval of 95 and a precision of 0.05 for a prevalence of $10 \%$. Hence within a period of six months, all pregnant women visiting the antenatal ward of the Teaching Hospital Peradeniya for blood sugar series, between 32 - 37 weeks of gestation were enrolled voluntarily to achieve the sample size necessitated. Written informed consent was obtained from all participants in the study, following adequate and necessary explanation.

Pregnant women with abnormal OGTT values $(\geq 5.6 \mathrm{mmol} / \mathrm{l}$ or 2 -hour value $\geq 7.8 \mathrm{mmol} / \mathrm{l}$ ), which is diagnostic for gestational diabetes (GDM) and those pregnant mothers who are previously diagnosed with type 1 or type 2 diabetes mellitus were enrolled to the study after obtaining informed consent. Pregnant women with normal values in 28-week OGTT and pregnant mothers who did not give consent were excluded from the study.

The blood samples were collected as described below. Patient preparation followed the common guidelines for Blood Sugar Series (BSS) testing. The women were advised to take their recommended meals and avoid aggressive physical activity before attending the postprandial blood sugar testing. The study population was divided into two groups, group 1 where the pregnant women's blood sugar control was managed with 1st-hour postprandial blood sugar values with a target to control blood sugar values $<7.8 \mathrm{mmol} / \mathrm{l}^{1}$ and group 2, managed with 2nd-hour values, with a target to control blood sugar values $<6.4 \mathrm{mmol} / \mathrm{l}^{1}$; the decision to follow up with $1^{\text {st }}$ hour or $2^{\text {nd }}$ hour was decided by the relevant consultant. Blood was drawn after 1-hour after meals in-group 1 in which control was achieved with 1st-hour values and 2 hours after meals in-group 2 where control was achieved with $2^{\mathrm{d}}$ hour values. Another Blood sample was collected at delivery for $\mathrm{HbAlc}$ analysis in both groups.

Standard pre-tested questionnaire (Appendix 1) designed by the authors 
and medical records were used to collect demographic data, BMI, age, gestation age of pregnant women and feto-maternal outcomes, the validity and reliability of the questionnaire was tested and confirmed after a pilot study. The feto-maternal outcomes were compared with the timings of postprandial glucose measurement and glycosylated haemoglobin values and mean values for HbA1c were calculated for each group. The Independent t-test was used to compare the means of both groups and Chi-square tests were used to analyse categorical data.

\section{Results}

\section{Basic characteristics of the study population}

A total of 69 pregnant women were recruited for group-1 where blood sugar control was optimized depending on the 1st-hour post-prandial blood glucose levels. The age range in this group was 18 to 42 years. There were $4.3 \%$ teenage pregnancies and the majority (40.2\%) comprised of women aged over 35 (Figure 1).

Another 69 pregnancies were used in group-2 where the blood sugar control was optimized depending on the 2nd-hour post-prandial blood glucose levels. The age range in this group was similar to group- 1 with $36.1 \%$ of women over the age of 35 and only $3 \%$ of teenage pregnancies (Figure 2 ).

A majority (44\%) of the women in group-1 belonged to the pre-obesity group [7] while $31 \%$ of the women were within the normal BMI range, with $5 \%$ of women categorised as underweight, $13 \%, 4 \%$ and $3 \%$ fell into obesity classes I, II and III respectively (Figure 3). In group-2 pre-obesity and normal weight groups [7] comprised of $38 \%$, while $6 \%$ of women were categorised as underweight, $12 \%, 4 \%$ and $3 \%$ fell into obesity classes I, II and III sequentially (Figure 4 ). In both groups a majority of women did not have pre-existing diabetes, only $23 \%$ had pre-exciting diabetes mellitus in group- 1 and $27 \%$ in group- 2 .

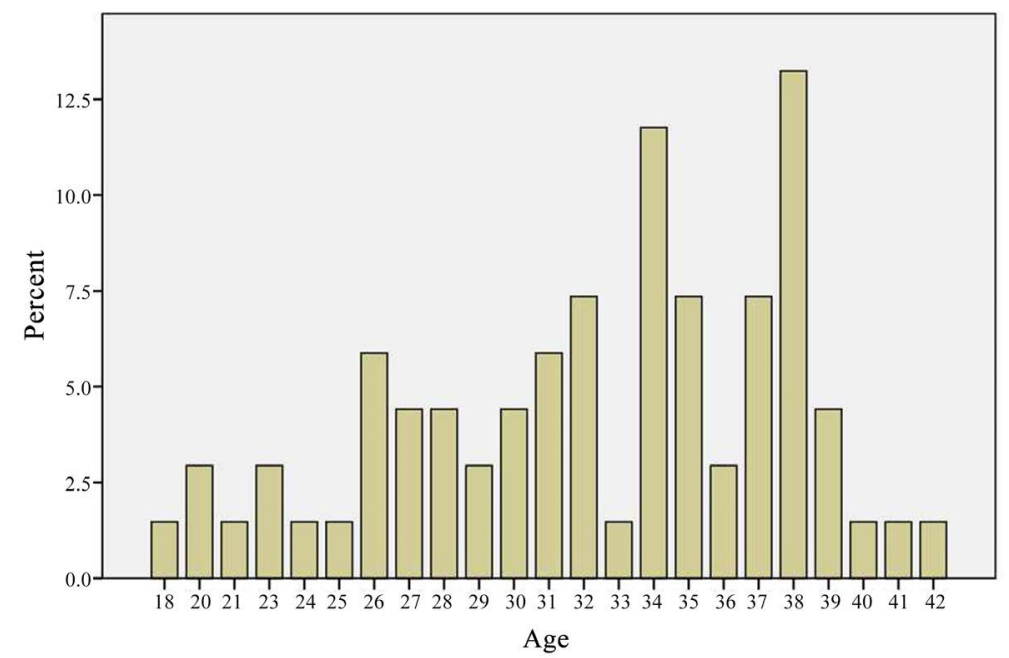

Figure 1. Maternal age of the group controlled with $1^{\text {st }}$-hour postprandial blood sugar values. 


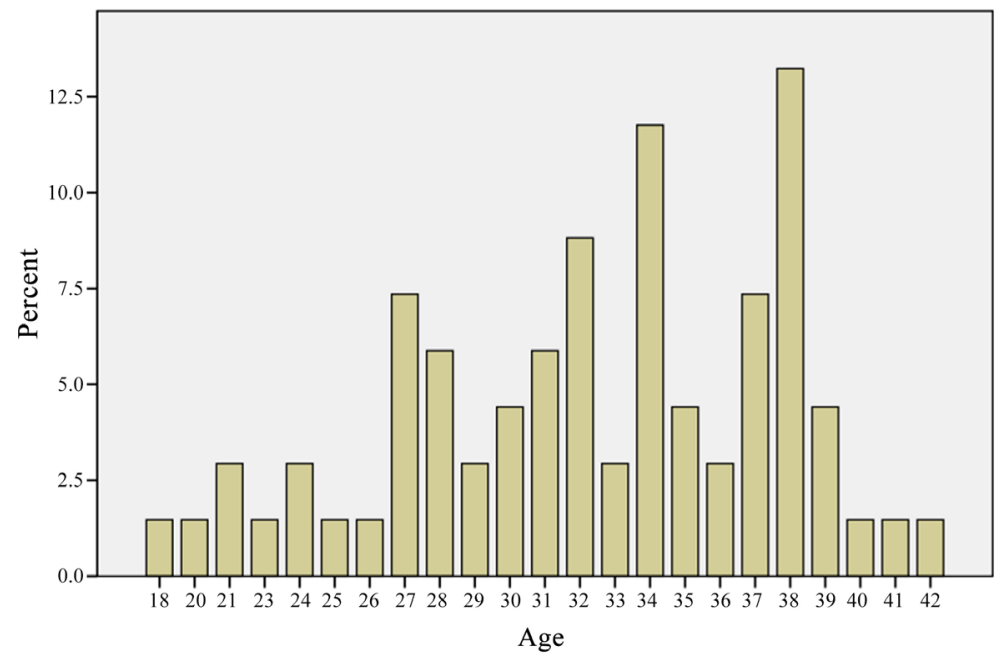

Figure 2. Maternal age of the group controlled with $2^{\text {nd }}$-hour postprandial blood sugar values.

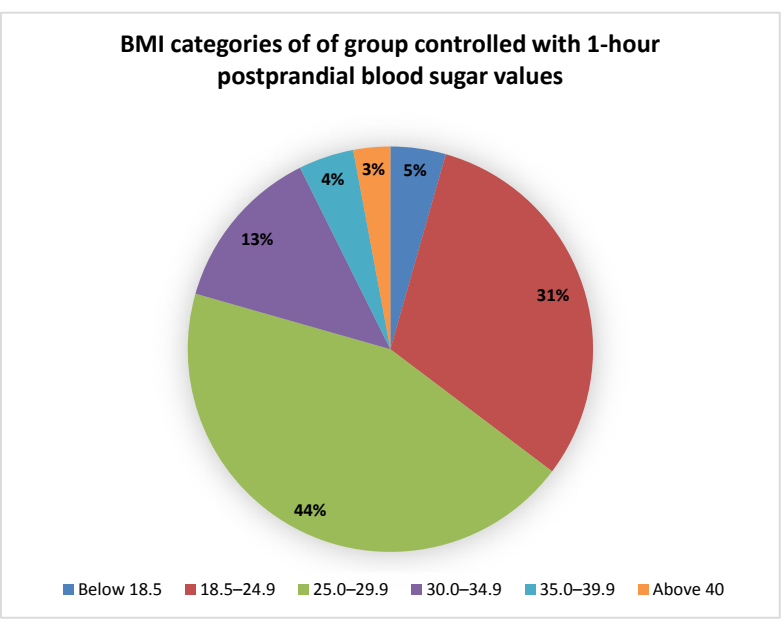

Figure 3. BMI (Body Mass Index) categories of the group controlled with $1^{\text {st }}$-hour postprandial blood sugar values.

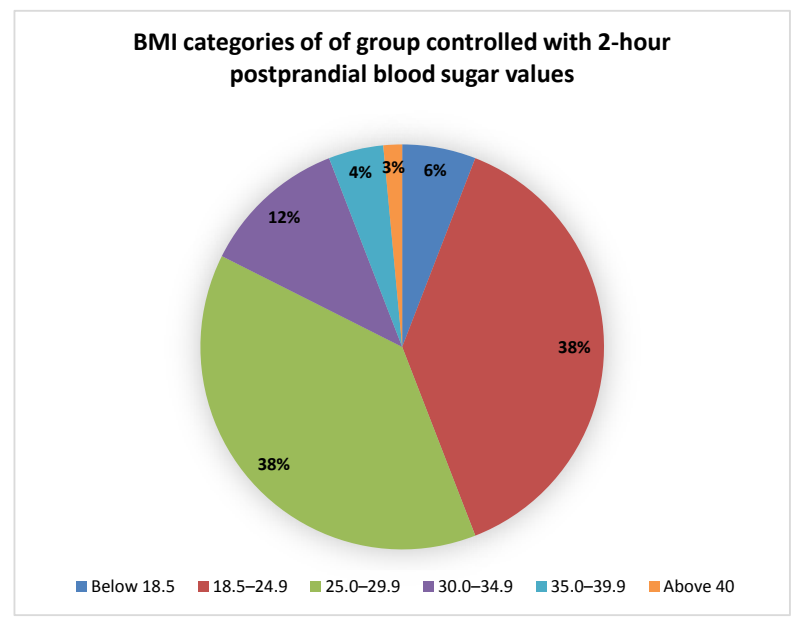

Figure 4. BMI (Body Mass Index) categories of the group controlled with $2^{\text {nd }}$-hour postprandial blood sugar values. 


\section{$H b A 1 c$ values in the study population}

Concerning women in group 1, the majority (53.03\%) were below the $\mathrm{HbAlc}$ of $5.7 \%(39 \mathrm{mmol} / \mathrm{mol})$ where control was satisfactory and the probability of having diabetes mellitus was low ${ }^{1}$. 40.91\% had HbAlc between 5.7\% - 6.4\% (39 $47 \mathrm{mmol} / \mathrm{mol}$ ) where the risk of developing diabetes mellitus was high. The remaining $6.06 \%$ had $\mathrm{HbAlc}$ above $6.5 \%$ ( $>48 \mathrm{mmol} / \mathrm{mol})$, these women were advised they had type 2 diabetes and referred for further care.

In group 2, 33.8\% were below the $\mathrm{HbA} 1 \mathrm{c}$ of $5.7 \%$ (39 $\mathrm{mmol} / \mathrm{mol}$ ) while $38.3 \%$ had HbAlc between 5.7\% - 6.4\% (39- $47 \mathrm{mmol} / \mathrm{mol}$ ). The remaining $27.9 \%$ had $\mathrm{HbAlc}$ of levels $6.5 \%$ ( $>48 \mathrm{mmol} / \mathrm{mol}$ ) or higher and similarly these women were referred for further care after counselling.

The difference of mean values of HbAlc obtained from the two groups was statistically significant $(\mathrm{p}=0.02)$ when analysed using the independent $\mathrm{t}$-test.

Delivery outcomes and feto-maternal complications in the study population

A large proportion (85.2\%) of the deliveries took place beyond $36^{+6}$ weeks of gestation in both groups and most of the deliveries occurred as normal vaginal deliveries, $57 \%$ in group- 1 and $54 \%$ in group-2 (Figure 5, Figure 6).

The LSCS rates $(p=0.735)$ were not statistically significant between the two groups when analysed using the chi-square test. Foetal birth weight ranged from $2 \mathrm{Kg}$ to $4.5 \mathrm{Kg}$ and foetal macrosomia [8] was detected in $6 \%$ of the neonates delivered in group 1, while $14.7 \%$ pregnancies ended up with low birthweight babies [9]. Group 2 also had the same foetal birth weight range. However, $10 \%$ of the neonates in group 2 had macrosomia and 10.3\% neonates had a low birth weight. The occurrence of foetal macrosomia was significantly $(\mathrm{p}<0.01)$ low in the one-hour postprandial blood sugar control group when analysed using the chi-square test. There were no reported stillbirths in both groups during the study period while the shoulder dystocia occurrence was $1.4 \%$ in group- 1 and

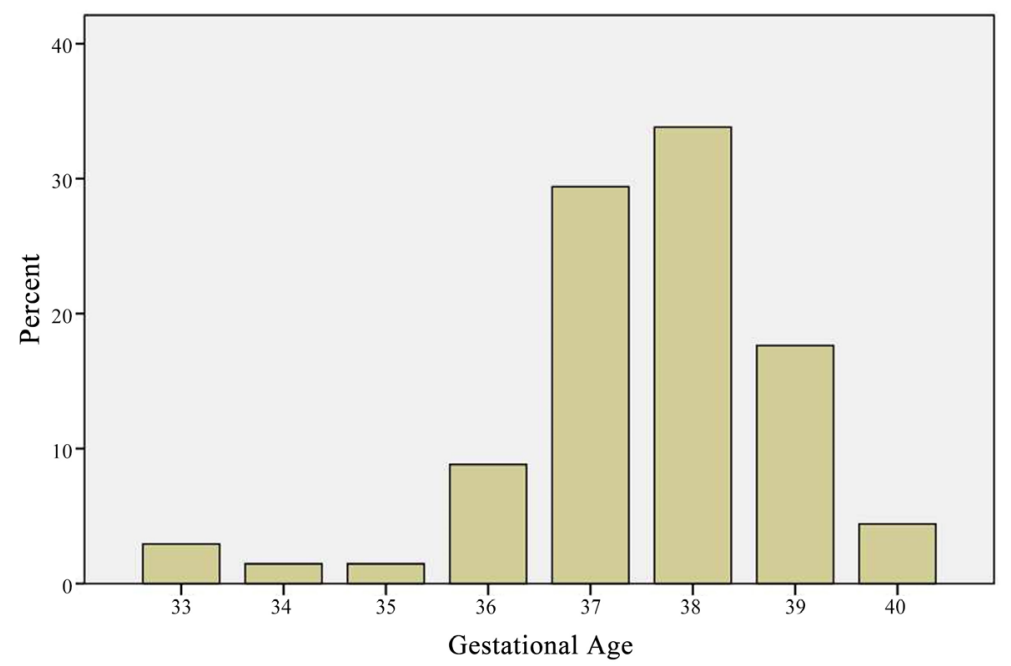

Figure 5. Gestational age at delivery of the group controlled with $1^{\text {st }}$-hour postprandial blood sugar values. 


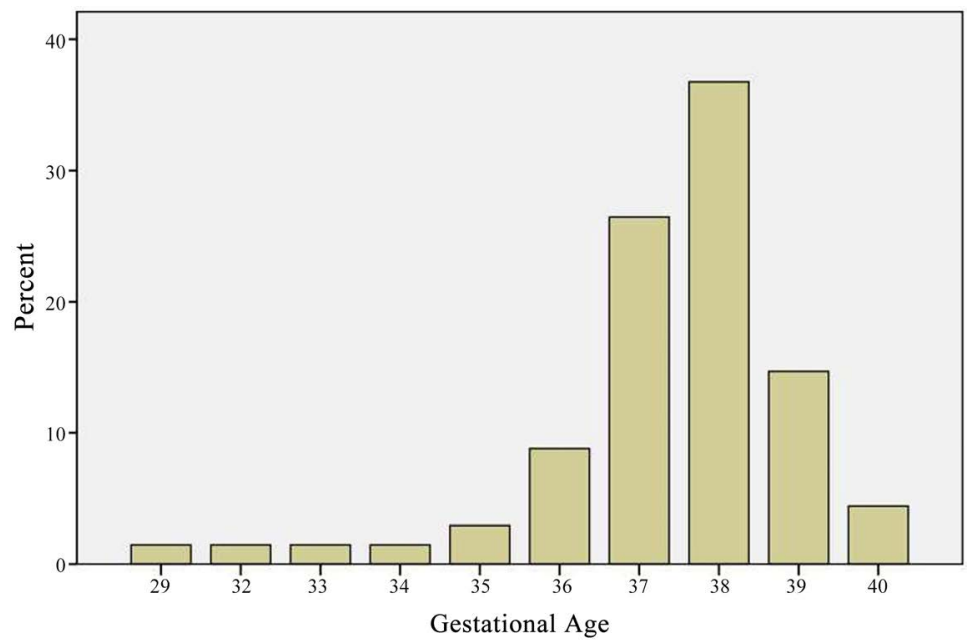

Figure 6. Gestational age at delivery of the group controlled with $2^{\text {nd }}$-hour postprandial blood sugar values.

$5.8 \%$ in group 2, the occurrence of shoulder dystocia was not statistically significant $(\mathrm{p}=0.062)$ nonetheless. Hypoglycaemic episodes were experienced by $6 \%$ of neonates e in group - 1 and by $8 \%$ in group 2 , yet the difference was not statistically significant $(\mathrm{p}=0.2126)$. Furthermore, the occurrence of congenital malformations was $4 \%$ and $3 \%$ in group- 1 and group- 2 respectively with a statistically insignificant difference $(\mathrm{p}=0.208)$. Lastly, during the follow up $7.4 \%$ of pregnant mothers were reported to have developed pre-eclampsia in group-1 and $8.8 \%$ in group- 2 . Nevertheless, the difference was not statically significant.

\section{Discussion}

\section{Role of $\mathrm{HbA1c}$ in the assessment of glycaemic control in pregnancy}

The National Institute for Health and Care Excellence has introduced target ranges (Fasting blood sugar, and targets for $1^{\text {st }}$ and 2 nd-hour postprandial) for blood glucose monitoring during pregnancy. ${ }^{1}$ However, due to practical reasons, it is difficult to follow up and optimize blood glucose levels using all three recommended values. This gives rise to the question, as to whether the use of the $1^{\text {st }}$ or 2nd-hour values is more advantageous with regards to glucose optimization during pregnancies.

According to Chaudry, R et al. [10], during pregnancy the HbAlc reflects the glycaemic control over the last 6 to 8 weeks, owing to shortened life span and increased turnover rate of red blood cells during the pregnancy. Therefore, in comparison to a non-pregnant state where the HbAlc reflects the mean blood glucose concentration of the preceding 8 - 12 weeks, its interpretation is adversely affected by the pregnancy state. However, it can be used to assess the preceding 6 8 weeks of the delivery, which is a critical period where both the foetus and the mother are susceptible to adverse pregnancy outcomes.

There are other serum markers which undergo non-enzymatic glycation, providing end glycosylation products enabling measurements that reflect one's glycaemic control. For example, a good correlation was noted with fructosamine 
levels with that of $\mathrm{HbAlc}$ yet, an inter-personal variation of fructosamine was comparatively higher than $\mathrm{HbAlc}$, hence, a much larger increment is needed for a significant change to occur [10]. As the turnover rate is higher for proteins bound to albumin compared to haemoglobin (28 compared to 120 days for haemoglobin) the fructosamine levels reflect mean blood glucose over the last 1 2 weeks only [10]. Therefore, in this clinical setting HbAlc was considered as the investigation of choice.

\section{Epidemiological factors of the study population}

In terms of age distribution, a significant proportion of the participants were recognised as over 35 years and these pregnant women were susceptible to the risk of developing gestational diabetes due attributes such as their older age and their South-Asian ethnicity.

Those who were already being diagnosed with pre-existing diabetes, as a consequence of being diabetic for an extended period, were deemed more vulnerable to both microscopic and macroscopic complications. [11] and in turn, were probable to experience adverse pregnancy outcomes [12]. The prevalence of pre-existing diabetes is nearly equivalent in both groups and it is presumed to not have contributed to any bias towards the study results. Similarly, the gestational age which the majority of the deliveries occurred was also compatible in both groups. Therefore, when comparing the perinatal outcomes of the two groups there were lesser chances of bias against one group.

\section{$\mathrm{HbA1c}$ in $1^{\text {st }}$ hour versus $2^{\text {nd }}$ hour postprandial blood sugar optimization groups}

The results depict a significantly lower $(\mathrm{p}=0.02)$ mean $\mathrm{HbA1c}$ value of $5.7284 \%$ for the 1st-hour postprandial glucose optimization group (Table 1) compared to the 2nd-hour control group. Therefore, during the preceding 6 - 8 weeks of delivery, the overall control of blood sugar values was superior in the group where the treatment was adjusted based on 1st-hour postprandial glucose values.

This effect was seen even though the 1st-hour postprandial blood sugar optimization group had a predominant number of pregnant women classified under the pre-obesity category and a small minority of pregnant women with a normal BMI in contrast to the 2nd-hour blood sugar optimisation group.

Since many pregnancy complications (large for gestational age, stillbirths, shoulder dystocia during delivery etc.) occur during the second half of the pregnancy [12] we have concentrated mainly on the optimisation of blood sugar control during the latter half of the pregnancy.

Table 1. HbA1c values of $1^{\text {st }}$ and 2 nd-hour postprandial blood sugar control group.

\begin{tabular}{cccccc}
\hline & Group & N & Mean & Std. Deviation & Std. Error Mean \\
\hline HbA1C & $\begin{array}{c}1^{\text {st }} \text {-hour postprandial blood } \\
\text { sugar control group }\end{array}$ & 69 & 5.7284 & 0.47259 & 0.05731 \\
HbA1C & $\begin{array}{c}2^{\text {nd }} \text {-hour postprandial blood } \\
\text { sugar control group }\end{array}$ & 69 & 6.0379 & 0.63508 & 0.07701 \\
\hline
\end{tabular}


Feto-maternal outcomes in $1^{\text {st }}$ hour versus $2^{\text {nd }}$ hour postprandial blood sugar optimization groups

The occurrence of foetal macrosomia was significantly $(p<0.01)$ less in the 1st-hour postprandial blood glucose control group, these findings are in line with that of Combs, C et al., [13] further emphasising the importance of 1st-hour postprandial blood sugar optimization in glycaemic control in pregnancy. Post-natal hypoglycaemia is one of the most important metabolic complications related to hyperglycaemia in pregnancy, which should be anticipated and screened for [14]. In this study, the occurrence of hypoglycaemic episodes of newborns was relatively less (6\% compared to $8 \%$ ) in the 1st-hour blood sugar control group but the variance was not statistically different. Therefore, the occurrence of postnatal hypoglycaemia may not be affected by the timing of glucose optimization, but further research with larger sample size is required to conclude or refute this argument. Diabetic embryopathy causing the occurrence of congenital malformation occurs mainly before the seventh week of gestation where it is mainly affected by pre-gestational glycaemic control [1] [15]. However, the occurrence of congenital malformations was compared between the two groups and there was no significant difference. Moreover, the occurrence of these malformations was $<5 \%$ in both groups and it may indicate good pre-conception care for those with pre-existing diabetes mellitus. The findings of Holmes, V. et al. [16] demonstrated that women who ended up with pre-eclampsia had higher HbAlc values before and during the pregnancy period, suggesting the importance of optimal glycaemic control both before the pregnancy as well as throughout the pregnancy. During our research, there was no significant difference with the occurrence of pre-eclampsia among the two groups.

\section{Limitations}

In this study, we have taken into consideration the glycemic control of the preceding 6 - 8 weeks of the delivery with the aid of HbAlc. Therefore, it limits our ability to make conclusions about the entire pregnancy period about glycemic control and some of its effects. For example, we cannot get an idea about the blood sugar control concerning the second trimester and early third trimesters where there may be some fetal and maternal effects.

The indications of cesarean sections were not analyzed therefore we cannot conclude whether impaired glycemic control and its effects led to increasing cesarean deliveries or whether it is due to some other reason.

The ethnic differences, their lifestyle differences and patients perspective of the glycemic control was not addressed, which is an important factor before implementing guidelines to relevant societies.

\section{Conclusions}

Long-term control of blood sugar was significantly superior among the subjects managed with 1st-hour postprandial blood sugars with statistically significant 
favourable $\mathrm{HbAlc}$ results and reduced the occurrence of fetal macrosomia.

Further research is needed to assess the glycaemic control through the pregnancy, 1st-hour blood glucose optimisation to that of 2nd-hour blood glucose optimisation. Besides, in detail analysis of fetal effects and caesarean section indication should be addressed by future research.

\section{Funding}

No funds.

\section{Acknowledgements}

I would like to thank Prof. S.U.B. Thennakoon, Professor in Community Medicine, Department of Community Medicine, for the statistical, for the advice given on statistical analysis of the data \& Ms. M.R.S. Muhandirum Research assistant, Department of Obstetrics and Gynaecology, Faculty of Medicine, the University of Peradeniya for assisting data collection.

\section{Conflicts of Interest}

The authors declare no conflicts of interest regarding the publication of this paper.

\section{References}

[1] NICE (2015) Diabetes in Pregnancy: Management from Preconception to the Postnatal Period. 1-Recommendations. Guidance and Guidelines. NICE.

[2] Kinsley, B. (2007) Achieving Better Outcomes in Pregnancies Complicated by Type 1 and Type 2 Diabetes Mellitus. Clinical Therapeutics, 29, S153-S160. https://doi.org/10.1016/j.clinthera.2007.12.015

[3] Nolan, C.J. (2011) Controversies in Gestational Diabetes. Best Practice and Research: Clinical Obstetrics and Gynaecology, 25, 37-49. https://doi.org/10.1016/j.bpobgyn.2010.10.004

[4] Weisz, B., et al. (2005) One Hour versus Two Hours Postprandial Glucose Measurement in Gestational Diabetes: A Prospective Study. Journal of Perinatology, 25 241-244. https://doi.org/10.1038/sj.jp.7211243

[5] Ben-Haroush, A., et al. (2004) The Postprandial Glucose Profile in the Diabetic Pregnancy. American Journal of Obstetrics and Gynecology, 191, 576-581. https://doi.org/10.1016/j.ajog.2004.01.055

[6] Gruendhammer, M., Brezinka, C. and Lechleitner, M. (2003) The Number of Abnormal Plasma Glucose Values in the Oral Glucose Tolerance Test and the Feto-Maternal Outcome of Pregnancy. European Journal of Obstetrics \& Gynecology and Reproductive Biology, 108, 131-136. https://doi.org/10.1016/S0301-2115(02)00370-6

[7] Euro.who.int. (2018) Body Mass Index BMI. http://www.euro.who.int/en/health-topics/disease-prevention/nutrition/a-healthy-li festyle/body-mass-index-bmi

[8] Ng, S.K., Olog, A., Spinks, A.B., Cameron, C.M., Searle, J. and McClure, R.J. (2010) Risk Factors and Obstetric Complications of Large for Gestational Age Births with 
Adjustments for Community Effects: Results from a New Cohort Study. BMC Public Health, 10, 460. https://doi.org/10.1186/1471-2458-10-460

[9] Royal College of Obstetricians \& Gynecologists (2013) Small-for-Gestational-Age Fetus, Investigation and Management (Green-Top Guideline No. 31).

https://www.rcog.org.uk/en/guidelines-research-services/guidelines/gtg31

[10] Chaudry, R., Gilby, P. and Carroll, P. (2007) Pre-Existing (Type 1 and Type 2) Diabetes in Pregnancy. Obstetrics, Gynaecology \& Reproductive Medicine, 17, 339-344. https://doi.org/10.1016/j.ogrm.2007.09.001

[11] Zoungas, S., Woodward, M., Li, Q., Cooper, M., Hamet, P., Harrap, S., Heller, S., Marre, M., Patel, A., Poulter, N., Williams, B. and Chalmers, J. (2014) Impact of Age, Age at Diagnosis and Duration of Diabetes on the Risk of Macrovascular and Microvascular Complications and Death in Type 2 Diabetes. Diabetologia, 57, 2465-2474. https://doi.org/10.1007/s00125-014-3369-7

[12] Buchanan, T., Xiang, A. and Page, K. (2012) Gestational Diabetes Mellitus: Risks and Management during and after Pregnancy. Nature Reviews Endocrinology, 8, 639-649. https://doi.org/10.1038/nrendo.2012.96

[13] Combs, C., Gunderson, E., Kitzmiller, J., Gavin, L. and Main, E. (1992) Relationship of Fetal Macrosomia to Maternal Postprandial Glucose Control during Pregnancy. Diabetes Care, 15, 1251-1257. https://doi.org/10.2337/diacare.15.10.1251

[14] Stanescu, A.S. (2014) Neonatal Hypoglycemia Screening in Newborns from Diabetic Mothers-Arguments and Controversies. Journal of Medicine and Life, 7, 51-52. https://www.ncbi.nlm.nih.gov/pmc/articles/PMC4391423

[15] Chen, C. (2005) Congenital Malformations Associated with Maternal Diabetes. Taiwanese Journal of Obstetrics and Gynecology, 44, 1-7.

[16] Holmes, V., Young, I., Patterson, C., Pearson, D., Walker, J., Maresh, M. and McCance, D. (2011) Optimal Glycemic Control, Pre-Eclampsia, and Gestational Hypertension in Women with Type 1 Diabetes in the Diabetes and Pre-Eclampsia Intervention Trial. Diabetes Care, 34, 1683-1688. https://doi.org/10.2337/dc11-0244 


\section{Appendix 1}

\section{Questionnaire}

Ref No:

Comparison of postprandial blood glucose $1 \mathrm{~h}$ VS. $2 \mathrm{~h}$

\section{Patients Record}
1) Patients Name:
2) Age:
3) Date of Birth:
4) Occupation:
5) Contact no:
6) Clinic No:

Gestational Age.

BMI.

Parity.

Pre-existing diabetes.

Oral glucose tolerance test values (OGTT).

Standard Laboratory venous plasma glucose values

\begin{tabular}{lll}
\hline & Group 1 $(1 \mathrm{~h})$ & Group 2(2 h) \\
\hline Post breakfast & \\
Post lunch & \\
Post dinner & \\
\hline
\end{tabular}

\section{Postpartum maternal HbA1c value.}

\section{Feto-maternal outcome determination}

Fetal birth weight.

Head circumference.

Length....

Shoulder diameter.

\section{Complications of pregnancy}

Stillbirth

Congenital malformations

Macrosomia

Birth injury

Postnatal adaptation problems (Hypoglycemia)

pre-eclampsia

Caesarean section 